\title{
Cuidado ao câncer e a prática interdisciplinar
}

\author{
Cancer care and interdisciplinary practice
}

\author{
Cuidado del cáncer y la práctica interdisciplinaria
}

TRATADO DE ENFERMAGEM EM ONCOLOGIA. Silva RCV, Sant'Ana RSE, Cardoso MBR, Alcântara LFFL, organizadores. Lisboa: Chiado Books; 2018. Volume I, 698 p. ISBN 978-98952-2920-8. Volume II, 444 p. ISBN 978-989-522923-9.

doi: 10.1590/0102-311X00193218

As transições demográficas e epidemiológicas globais sinalizam um impacto cada vez maior da carga de câncer nas próximas décadas 1 . Configurando como uma das principais causas de mortalidade mundial em países de todos os níveis de renda, o câncer cursa com elevado ônus psicossocial e econômico e é considerado como um importante problema global de saúde pública 1. Dados da última estimativa do Instituto Nacional de Câncer José Alencar Gomes da Silva (INCA) apontam para o biênio 2018-2019 a ocorrência de 600 mil casos novos de câncer, para cada ano 2 .

O cuidado ao paciente com câncer tem tido uma evolução exponencial nas técnicas diagnósticas e terapêuticas, contribuindo para uma maior sobrevida e qualidade de vida dessa população. Cabe aos profissionais de saúde acompanhar o desenvolvimento desse cenário complexo de cuidado por meio das investigações científicas, que são os principais recursos para a atualização do conhecimento para o cuidado perso- nalizado ao paciente oncológico. No contexto do câncer, os profissionais de saúde que compõem a equipe multiprofissional apresentam como competências essenciais: prestar assistência, atuar na prevenção, controle, avaliação diagnóstica, tratamento, reabilitação e atendimento aos familiares; além de desenvolverem ações educativas e ações integradas com outros setores da sociedade. A pesquisa na área do cuidado ao câncer é essencial para gerar a base de conhecimento que fundamenta a prática clínica e as políticas públicas neste campo, além de poder identificar o impacto do câncer e do tratamento na vida de pacientes e familiares 3 . Os profissionais de saúde, incluindo os enfermeiros, têm buscado desenvolver métodos e abordagens, protocolos clínicos e intervenções efetivas às reais necessidades dos pacientes com câncer e seus familiares, com vistas ao cuidado personalizado 3,4 .

Um aspecto relevante a se destacar no cuidado ao câncer se refere à definição conceitual dos tipos de equipes existentes: multidisciplinar, interdisciplinar. Multidisciplinaridade é definida como um conjunto de disciplinas que trata, simultaneamente, de uma dada questão, sem que os profissionais implicados estabeleçam efetivas trocas entre si 5 . Nesse caso, cada especialista emprega sua metodologia, baseado em suas hipóteses e teorias, sendo que o objeto em questão é visto sob múltiplos pontos de vista, numa justaposição de conhecimentos, ou seja, o mesmo tema é abordado sob ângulos variados, não existindo a perspectiva de síntese. Interdisciplinaridade 
detém o caráter "inter”, marcando a presença de uma ação recíproca de um elemento sobre o outro e vice-versa. Em uma equipe interdisciplinar há possibilidade de troca de instrumentos, técnicas, metodologia e abordagens entre as disciplinas. Trata-se de um diálogo que leva ao enriquecimento e transformação das disciplinas envolvidas 5 , compreendendo-se o estabelecimento de relações menos verticais entre as disciplinas. Nesse caso, não há uma justaposição entre os elementos disciplinares, mas, sim, uma combinação nova de elementos internos, com trocas entre os saberes para uma tarefa ser realizada em conjunto. Ademais, a interdisciplinaridade traz reflexões e contribuições importantes para a produção científica contemporânea, na medida em que contempla as intersubjetividades e o respeito às diferenças existentes diante de múltiplos olhares e compreensões acerca de um determinado objeto ${ }^{6}$, implicando uma consciência dos limites e das potencialidades de cada campo do saber, na busca de um fazer coletivo, como deve ser o cuidado ao câncer.

Ressalta-se que inúmeros e complexos desafios permeiam a prática interdisciplinar do cuidado personalizado à pessoa com câncer e à sua família 3. Dessa forma, acolher e valorizar a participação autônoma e colaborativa nas tomadas de decisões compartilhadas na atenção ao câncer demandam dos profissionais de saúde o desenvolvimento de competências, habilidades, atitudes, métodos e abordagens que abarquem as necessidades de assistência, gestão e pesquisa, de modo a primar por um cuidado de qualidade. Nesse sentido, a publicação Tratado de Enfermagem em Oncologia, organizada por Rita de Cássia Veloso da Silva, Ricardo Souza Evangelista Sant'Ana, Marlize Brandão Ribeiro Cardoso \& Laisa Figueiredo Ferreira Lós de Alcântara, lançada em agosto de 2018 pela editora Chiado Books (Lisboa, Portugal), possibilita novos conhecimentos e reflexões acerca do cuidado interdisciplinar ao câncer e da prática profissional de enfermagem em Oncologia 7.

O Tratado é um compilado de textos escritos por 128 pesquisadores, especialistas na área de cuidado ao câncer, predominantemente do Brasil e de Portugal, e conta com pesquisadores do Canadá e da Espanha. São pesquisadores de universidades e centros de referência nacionais e internacionais, renomados em Oncologia e com larga experiência na temática. A organização do Tratado está ancorada na tríade: assistência, gestão/educação e pesquisa em Oncologia. A publi- cação é dividida em dois volumes, totalizando 40 capítulos. O volume I conta com 25 capítulos que abrangem aspectos relacionados à atenção à pessoa com câncer e a seus cuidadores sob a perspectiva interdisciplinar. Esses capítulos aprofundam tópicos atinentes da assistência ao paciente oncológico, tais como: atenção à família da pessoa com câncer; cuidados paliativos; problemas éticos vivenciados no cuidado à pessoa com câncer; equipe de saúde na comunicação com o paciente oncológico; burnout em profissionais de saúde em Oncologia; sexualidade e câncer; espiritualidade no cuidado em Oncologia; sobreviventes ao câncer; luto antecipatório e final; quimioterapia e biossegurança na manipulação de quimioterápicos; radioterapia; transplante de células-tronco hematopoiéticas; manejo seguro de acessos vasculares centrais e periféricos; cuidados às pessoas com feridas neoplásicas malignas; manejo da dor em Oncologia; o câncer no homem; suporte terapêutico complementar em Oncologia; delirium, desidratação em pacientes oncológicos; e atenção à fadiga no contexto de cuidados paliativos

O volume II do Tratado é constituído por outros 15 capítulos distribuídos em dois grandes eixos: "educação e gestão em oncologia" (composto por nove capítulos) e "pesquisa em oncologia" (composto por seis capítulos). No eixo "educação e gestão em oncologia” são apresentados os seguintes temas: o enfermeiro educador extramuros; ações da enfermagem na prevenção e no controle do câncer; atuação da enfermagem no setor de diagnóstico por imagem; competências do enfermeiro em oncohematologia; ações da enfermagem na radioiodoterapia; segurança do paciente na terapêutica oncológica; indicadores de qualidade em Oncologia; acreditação em Oncologia. No eixo "pesquisa em oncologia" temas inovadores são resgatados e aprofundados, tais como: pesquisa de enfermagem em Oncologia; Oncologia integrativa; aconselhamento genético em câncer; psiconeuroimunologia e câncer; e pesquisa translacional em Oncologia.

A leitura dos capítulos é prazerosa, uma vez que os autores utilizam os recursos de tabelas e figuras para destacar ou resumir informações importantes, e também oferecem uma atualizada lista de referências para que o leitor interessado possa consultar. Todos os capítulos começam elencando os seus objetivos, seguidos por um sumário, resumo e considerações iniciais. Tal estrutura permite ao leitor apreender os principais pontos a serem desenvolvidos no corpo de 
cada capítulo. Ao final de cada um também existe uma seção de implicações para a prática clínica no cuidado ao câncer e conclusão, possibilitando ao leitor registrar a informação essencial ou a mais relevante.

Esse Tratado impulsiona os profissionais de saúde a refletirem a respeito da prática interdisciplinar no cuidado ao câncer, repleta de desafios em busca da qualidade dos cuidados ofertados. Essa qualidade pressupõe a presença de profissionais que trabalhem em equipe interdisciplinar com alto grau de competência para o exercício profissional, de modo a otimizar esforços para a elaboração de projetos terapêuticos singulares; uso eficiente dos recursos; redução, a um nível mínimo, de danos produzidos ou decorridos da assistência; satisfação dos usuários quanto às suas demandas; e sobretudo, a um efeito favorável nos desfechos em saúde dessa população tão particular.

Embora a clínica, a arte de diagnosticar e tratar seus doentes não possa se desvincular completamente da especulação experimental, da investigação, que lhe fornece as evidências científicas para a prática profissional, de outro lado e tão importante quanto está o cuidado ao paciente com câncer que se beneficiará das descobertas destas investigações 3 . O cuidar é muito mais abrangente do que o curar e, que por vezes não necessita de sofisticações tecnológicas, mas sim de uma equipe interdisciplinar complexa, bem treinada e, sobretudo, humanizada 3,4. Nesse sentido, a abordagem interdisciplinar é essencial para o progresso no âmbito do cuidado ao câncer. Ademais, o sucesso dessa abordagem pode ser visto pela melhoria das curvas de sobrevida em todo o mundo, inclusive em diversos centros brasileiros.

Em adição, as propostas e diretrizes do Sistema Único de Saúde (SUS) defendem a necessidade de pensar o trabalho em equipe multiprofissional com vistas à interdisciplinaridade, contribuindo, desta forma, para a concretização da integralidade da atenção ${ }^{8}$. É justamente nesse sentido que o presente Tratado escrito para enfermagem pode contribuir para uma reflexão acerca de um pensamento para além da prática da corporação, mas que englobe os diversos atores do cuidado frente ao cenário complexo do câncer no Brasil, sob uma perspectiva interdisciplinar de modo a transformar a práxis.

Luis Carlos Lopes-Júnior 1

Regina Aparecida Garcia de Lima 1

1 Escola de Enfermagem de Ribeirão Preto, Universidade de São Paulo, Ribeirão Preto, Brasil.

lopesjr.lc@gmail.com

1. Siegel RL, Miller KD, Jemal A. Cancer statistics, 2018. CA Cancer J Clin 2018; 68:7-30.

2. Coordenação de Prevenção e Vigilância, Instituto Nacional de Câncer José Alencar Gomes da Silva. Estimativa 2018: incidência de câncer no Brasil. Rio de Janeiro: Instituto Nacional de Câncer José Alencar Gomes da Silva; 2017.

3. Lopes-Júnior LC, Olson K, de Omena Bomfim E, Pereira-da-Silva G, Nascimento LC, de Lima RA. Translational research and symptom management in oncology nursing. Br J Nurs 2016; 25:S12-21.

4. Lopes-Júnior LC, Bomfim EO, Nascimento LC, Nunes MD, Pereira-da-Silva G, Lima RA. Non-pharmacological interventions to manage fatigue and psychological stress in children and adolescents with cancer: an integrative review. Eur J Cancer Care (Engl) 2016; 25:921-35.

5. Almeida-Filho N. Transdisciplinaridade e o paradigma pós-disciplinar na saúde. Saúde Soc 2005; 14:30-50.

6. Follari R. Algumas considerações práticas sobre a interdisciplinaridade. In: Jantsch AP, Bianchetti L, organizadores. Interdisciplinaridade: para além da filosofia do sujeito. 6a Ed. Petrópolis: Editora Vozes; 2002. p. 97-110.

7. Silva RCV, Sant'Ana RSE, Cardoso MBR, Alcântara LFFL, organizadores. Tratado de enfermagem em oncologia. Lisboa: Chiado Books; 2018.

8. Peduzzi M. Equipe multiprofissional de saúde: conceito e tipologia. Rev Saúde Pública 2001; 35:103-9.

Recebido em 04/Out/2018

Versão final reapresentada em 15/Nov/2018

Aprovado em 21/Nov/2018 